\title{
To Unify or Not to Unify: A Case Study on Unified Builds (in WebKit)
}

\author{
Takafumi Kubota \\ Keio University \\ Japan \\ takafumi@sslab.ics.keio.ac.jp
}

\author{
Yusuke Suzuki \\ Keio University \\ Japan \\ yusuke.suzuki@sslab.ics.keio.ac.jp
}

\author{
Kenji Kono \\ Keio University \\ Japan \\ kono@sslab.ics.keio.ac.jp
}

\begin{abstract}
Unified builds are a simple but effective technique to reduce the build time of large software projects. Unified builds generate large compiler tasks by bundling multiple source files into one, resulting in a significant reduction in build time through removal of redundant work incurred by shared headers. However, unified builds have a negative effect on incremental builds because each compiler task gets larger. An ad-hoc unification strategy causes an excessive slowdown in incremental builds. A rough report from WebKit says the worst slowdown is $20 \%$ ( $6 \mathrm{~s} \rightarrow 7 \mathrm{~s}$ ), but our investigation shows it is as high as $479 \%(19 \mathrm{~s} \rightarrow 110 \mathrm{~s})$.

In this paper, we investigate the characteristics of unified builds to find a sweet spot, which generates compiler tasks that reduce the full build time without increasing the incremental build time. An indepth analysis of WebKit reveals 1) source files with higher header similarity should be unified, 2) source files that have significant differences in compile times should not be unified, and 3) source files that are not frontend-intensive should not be unified. Our case study shows the total build time is reduced by $2.66 \%$, and the worst slowdown falls from $479 \%$ to $129 \%$. These findings will be of help in deriving a more intelligent strategy of unification and give a basis for discussions on future build systems, compilers, and module systems that cooperatively generate efficient compiler tasks.
\end{abstract}

\section{CCS CONCEPTS}

- Software and its engineering $\rightarrow$ Development frameworks and environments; Compilers; Software configuration management and version control systems;

\section{KEYWORDS}

Unified builds, Incremental builds, Build system, Source code characterization, Compile time analysis

\section{ACM Reference Format:}

Takafumi Kubota, Yusuke Suzuki, and Kenji Kono. 2019. To Unify or Not to Unify: A Case Study on Unified Builds (in WebKit). In Proceedings of the 28th International Conference on Compiler Construction (CC '19), February 16-17, 2019, Washington, DC, USA. ACM, New York, NY, USA, 11 pages. https://doi.org/10.1145/3302516.3307347

Permission to make digital or hard copies of all or part of this work for personal or classroom use is granted without fee provided that copies are not made or distributed for profit or commercial advantage and that copies bear this notice and the full citation on the first page. Copyrights for components of this work owned by others than ACM must be honored. Abstracting with credit is permitted. To copy otherwise, or republish, to post on servers or to redistribute to lists, requires prior specific permission and/or a fee. Request permissions from permissions@acm.org.

CC '19, February 16-17, 2019, Washington, DC, USA

(C) 2019 Association for Computing Machinery.

ACM ISBN 978-1-4503-6277-1/19/02 ..\$15.00

https://doi.org/10.1145/3302516.3307347

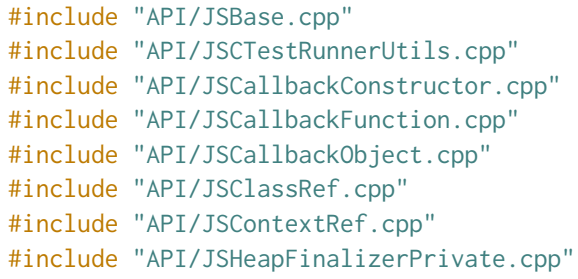

Figure 1. Example of unified source files in WebKit.

\section{INTRODUCTION}

As the code size of software projects has increased, the build time of software projects that use compiled languages has become a headache. Long build times slow down the development cycle and the time taken for a build crucially affects the productivity of developers, who are idle during the build [31]. According to our investigation of the logs of the WebKit build bot [16], 1,387 build tasks were executed from March 17 to August 3 in 2018. The build bot was active 75 days, and builds that took more than 15 minutes occurred 164 times on 61 out of 75 days. In these long builds, the number of recompiled source files was 1,030 on average.

Traditionally, build systems have been used to make builds more efficient and accelerate the development cycle. The primary advantage of build systems (e.g., GNU Make [10], CMake [4], Ninja [12], and Meson [13]) is incremental builds, which manage the dependencies among source files and recompile only the updated files and those dependent on them. Build systems are evolving to meet the increasingly complicated demands of software projects; the first version of CMake, which is a higher-level build system, was released in 2000 and supports flexible configurations. Ninja was released in 2012 and is designed to support minimal features for very fast incremental builds intended to be used in higher-level build systems. Recently, compiler caching tools such as CCache [2] and cHash [21] have been developed to speed up compilation of a single source file; previously compiled results are cached so that they can be re-used in the later compilation.

To shorten the build times of massive recompilations, some software projects have started to use unified builds (or unity builds). For example, WebKit [15] has adopted unified builds as its default build processing [28], and Chromium [3] supports unified builds in the form of Jumbo builds [8]. Unified builds are a simple but effective technique to speed up the compilation of multiple source files. They generate large compilation tasks by bundling multiple source files into one; Figure 1 shows an example of a "unified" file that merges eight files into one. 
This simple technique significantly reduces the build time for the following reasons. First, unified builds reduce redundant parsing of header files included multiple times from different source files. It is common for several source files to have identical headers because the header file often defines data structures, classes, and templates used in many source files. Second, especially in $\mathrm{C}++$, unified builds avoid redundant specializations of templates. Template specialization is a burden, because it instantiates all the bodies with template arguments specified in the source code. In unified builds, a compiler can reuse the specialized templates already instantiated in the source files in a unified file. As shown in Figure 1, when the last file (API/JSHeapFinlizerPrivate.cpp) is compiled, the compiler can trim redundant header parsing and template specializations that have already been done in the other seven source files. In WebKit, unified builds reduce the overall compilation time from 56m:05s to 26m:18s.

Despite these advantages, unified builds have an adverse effect on incremental builds. If a single file unified in the same unified file is updated, all the other files in that unified file have to be recompiled. This increases the rebuild time and diminishes the advantages of incremental builds. Our investigation of WebKit shows that incremental builds may slow down by as much as $479 \%$ $(19 \mathrm{~s} \rightarrow 110 \mathrm{~s})$. The WebKit's developer mailing list [22] is actively discussing the overhead caused by unified builds.

This paper sheds light on a unification strategy that can determine which source files should be bundled into a unified file. Current software projects, including WebKit [15] and Chromium [3], adopt an ad-hoc strategy; WebKit unifies up to eight source files in the same directory in alphabetical order, while Chromium bundles 50 files at a time on average by default. Our investigation shows these ad-hoc strategies are not optimal and there is room to reduce both the full build time and incremental build time. Our goal is to demonstrate there exists a sweet spot that generates compiler tasks that reduce the full build time and do not increase the incremental build time.

In summary, this paper makes two primary contributions:

- We perform an in-depth analysis of the unified build in WebKit as a case study and study the efficiency of the unified builds by answering the following research questions; RQ1: How does the similarity of the headers included among the source files affect unified builds?, RQ2: What combinations of source files are not suitable for unified builds?, RQ3: Where are the source files that can be unified?, and RQ4: How many source files should be merged into a unified source file?

- We also show empirical results that indicate our optimization for the unification strategy in WebKit improves the performance of the unified build. Our unification strategy in which the maximum bundle size is eight achieves a $2.66 \%$ speedup in the full build of WebKit while reducing the worst incremental build slowdown from $479 \%$ to $129 \%$.

The results of our analysis will be helpful for designing a more intelligent strategy for unification and give a basis for discussions on future build systems, compilers, and module systems that cooperatively generate efficient compiler tasks.

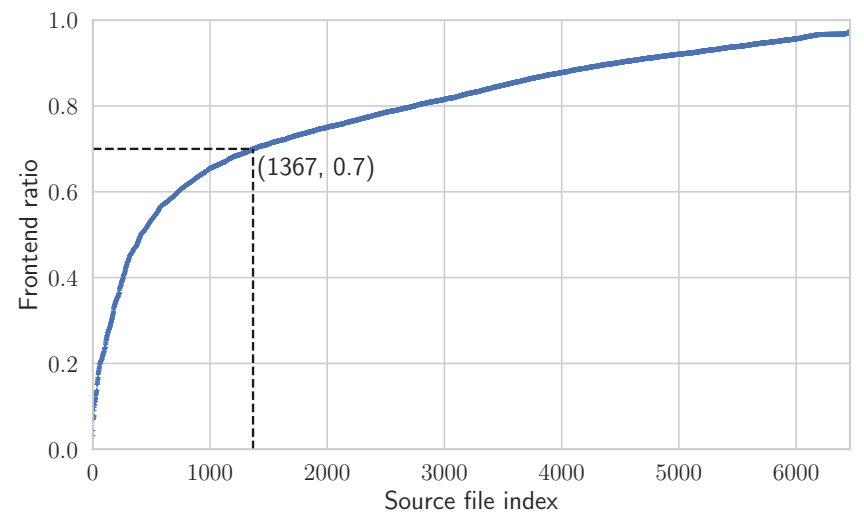

Figure 2. Frontend ratios of each compile time of 6,458 source files in WebKit. The ratio was measured using the timer report functionality of Clang (-ftime-report).

\section{BACKGROUND}

Speeding up sequential compilation is a key to the development of large software projects. Long build times occur almost every day, and they slow down the development cycle. Our analysis of the build bot logs of WebKit for GTK Linux 64-bit Release [16] shows that long-build tasks, entailing recompilation of more than 1,030 files and taking more than 15 minutes on average, were submitted on $81.3 \%$ of the days when the build bot was active. While distributed compiler infrastructures [5-7] mitigate long build times by parallelizing compiler tasks, they require a cluster of machines. Maintaining a cluster dedicated to building is costly and may be a barrier to contributors and users in open-source projects. Fast sequential compilation reduces the build time directly and is complementary to distributed compiler techniques.

To identify the bottleneck of the compilation, we decided to build WebKit as a large software project, measure the build time consumed in each source file, and characterize the breakdown of the build time. Figure 2 illustrates the frontend ratio of each file in its build time. The $\mathrm{x}$-axis is the proportion of the frontend to the entire compile time, and the $y$-axis is the files sorted by frontend ratio. The frontend mainly entails file reading, parsing, and C++ template specializations. The results show that the majority of source files are frontend-intensive. In $79 \%$ of the source files, the frontend of the compiler occupies more than $70 \%$ of the compile time. This high frontend ratio stems from the shared headers. Because multiple source files include these shared headers, the compiler repeatedly reads the shared headers, parses them, and instantiates template bodies across the source files.

\section{$2.1 \quad$ Unified Builds}

Unified builds remove the redundant frontend processing across multiple compiler tasks, resulting in a significant reduction in build time. Unified builds merge multiple compiler tasks into one. Since many of the source files likely include the same shared headers, an appropriately unified compiler task can remove redundant frontend processing of the shared headers. Figure 3 is an overview of unified builds. The build system merges source file $\mathrm{s} 1$ and $\mathrm{s} 2$ into one unified source file and generates the larger compiler task $\mathrm{s} 1+$ 


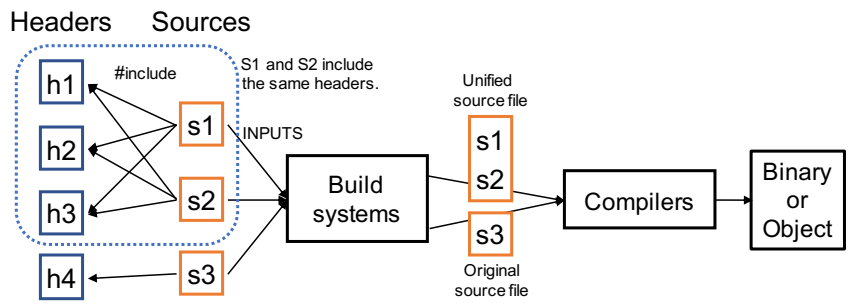

Figure 3. Overview of unified builds.

s2. Because s1 and s2 include the same shared headers, the unified compiler task s1 + s2 removes the redundant work of reading, parsing, and common template instantiation compared with compiling s1 and s2 separately. Some large projects such as WebKit [15] and Chromium [3] employ this technique [8,28], and it dramatically reduces the build time. These projects generate large compiler tasks by unifying source files with \#include directives, as shown in Figure 1 . This reduces the sequential build time dramatically, by $53 \%$ $(56 \mathrm{~m}: 05 \mathrm{~s} \rightarrow 26 \mathrm{~m}: 18 \mathrm{~s})$ in WebKit and by $66 \%(2 \mathrm{~h}: 33 \mathrm{~m}: 40 \mathrm{~s} \rightarrow 52 \mathrm{~m}: 24 \mathrm{~s})$ in Chromium.

The downside of unified builds is that they increase the build time of each compiler task, causing negative effects on incremental builds. Incremental builds track the updated source files and incrementally build updated ones from the previous build. Since unified builds enlarge each compiler task by bundling multiple source files, incremental builds of updated source files involve non-updated files bundled together. In Figure 3, when source file s1 is modified, the compiler has to recompile not only s 1 but also s2. This would be undesirable for developers who actively modify source code. The WebKit project reports that the worst slowdown is $20 \%(6 \mathrm{~s} \rightarrow 7 \mathrm{~s})$ in a typical scenario where one or two files are touched. Even worse, our extensive experiment described in Section 4.4 reveals that the overhead reaches $479 \%(19 \mathrm{~s} \rightarrow 110 \mathrm{~s})$.

\subsection{Motivation}

While unified builds are effective, there is as yet no quantitative analysis of them in large software projects. The lack of an in-depth analysis makes various aspects of unified builds unclear, preventing developers from using them in their projects, build systems, and compilers. One example of this situation is the trade-off between unified builds and incremental builds. Unified builds seem to have negative effects on incremental builds, but the build time numbers in practice have not been extensively analyzed.

Another problem relates to the strategy of unifying compiler tasks. Unifying appropriate source files is important to maximize the benefit of unified builds. However, projects have adopted unified builds with ad-hoc heuristics. For instance, WebKit unifies up to eight source files in the same directory, while Chromium bundles 50 files. The characteristics of the source files, such as the frontend ratio, are not considered in the unification process. Such ad-hoc strategies lead to sub-optimal build time gains and unnecessarily lengthen the build times of incremental builds.

This paper presents the first quantitative analysis of unified builds in a large software project. We investigate the effectiveness of unified builds in WebKit with various configurations and identify the characteristics affecting the above-mentioned trade-off. On the basis of these findings, we demonstrate that our unification strategy achieves build-time speedups without slowing down incremental builds.

The concept of unified builds is to unify multiple compiler tasks into one efficient compiler task. This paper focuses on the approach of unifying source files with \#include directives, because it is used in the wild. Although this approach looks like a workaround, our findings can be applied to future build systems and language supports that leverage unified builds in a non-ad-hoc manner.

\section{STUDY}

This section describes the research questions and defines the metrics.

\subsection{Research Questions}

We will explore five research questions in an attempt to reveal the unclear heuristics in making the decision to unify or not to unify source files:

\section{- RQ1: How does the similarity of the headers included} among the source files affect unified builds? As described in the previous section, unified builds reduce redundant work of the shared headers included in multiple source files. Our hypothesis is that unified builds work well when the included headers overlap among the source files. Here, we show how the similarity of the included headers affects unified builds. These findings help us to understand the potential impact of the header similarity on unified builds.

- RQ2: What combinations of source files are not suitable for unified builds? We find cases where unified builds perform poorly and identify two kinds of source file combination that have negative effects on unified builds. These findings help us to avoid such source file combinations when building unify rules and improve the efficiency of unified builds.

- RQ3: Where are the source files that can be unified? Currently, WebKit only unifies source files in the same directory. However, in practice, there are many cases in which source files in different directories would be suitable for unified builds. These findings increase the opportunities to unify such source files.

- RQ4: How many source files should be bundled into a unified source file? The bundle size is critical to compiling speedups and incremental build slowdowns. Unifying too many source files causes unacceptable slowdowns in incremental builds. On the other hand, unifying only a few source files does not lead to compile-time speedups. To better understand this trade-off, we analyze the compile time when using unified builds for three different kinds of source files while changing the unified size. These findings help us to identify the best bundle size depending on the characteristics of each source file.

- RQ5: How can we improve unified builds (in WebKit)? Lastly, we perform an empirical study on optimizing unified builds in WebKit. We tune the unify rule of WebKit with the findings of RQ1 $\sim$ RQ4 and compare it with the original unify rule. We measure the efficiency of unified builds in terms of the number of generated unified source files, full build time, and 


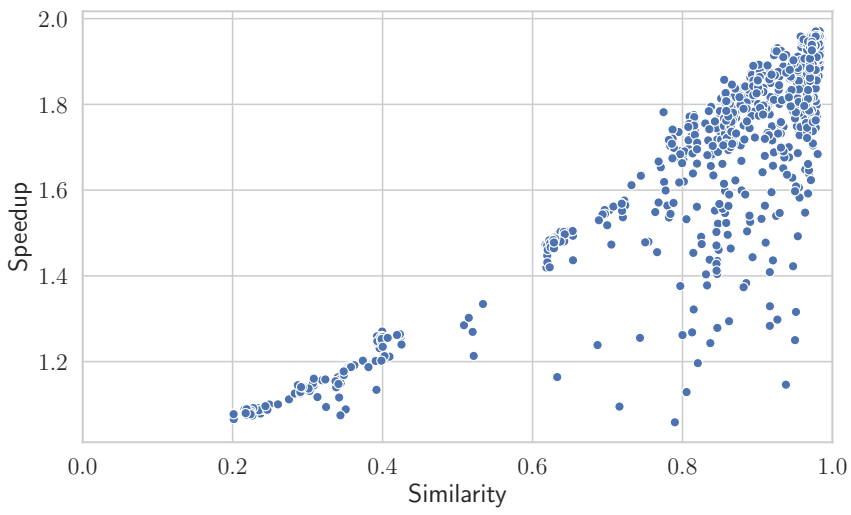

(a) Compile-Time Speedup (higher is better).

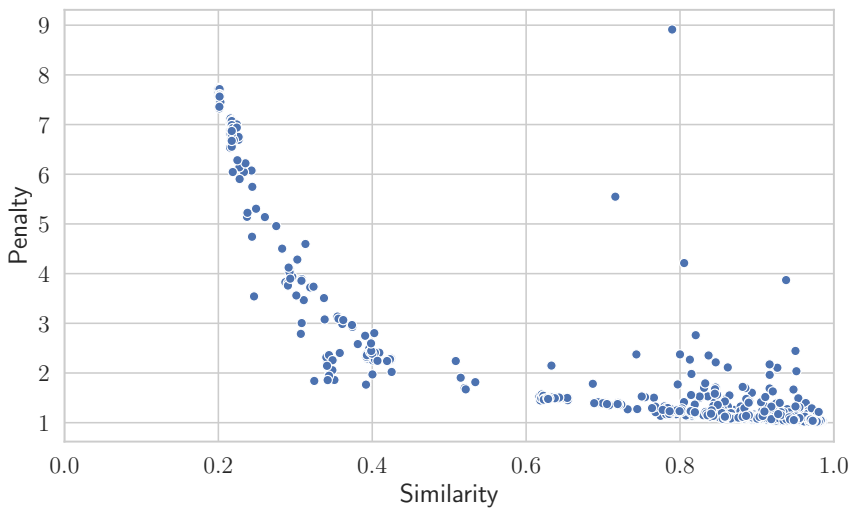

(b) Expected Incremental Build Penalty (lower is better).

Figure 4. The figures show the impact of the similarity of the included headers on the unified build. The impact on the unified build of API/JSBase.cpp in combination with each of 959 different source files in JavaScriptCore of WebKit is shown. Each dot indicates a pair of the unified source files. The $x$-axes of the figures depict the similarity between API/JSBase.cpp and the paired source file. The $y$-axis shows the impact on the unified build; speedup in Figure $4 a$ and penalty on the incremental build in Figure $4 \mathrm{~b}$.

slowdown of incremental builds. We also evaluate the performance of the output binaries. The results highlight the need for a sophisticated strategy to adjust the trade-offs between unified builds and incremental builds.

\subsection{Metrics}

The following metrics are used in the analysis.

- Header Similarity. The similarity between two source files is quantified by the extent that the same headers are included. The Jaccard index is used as the measure of similarity, which is defined as the size of the intersection divided by the size of the union of two sets. Thus, the similarity of two source files is determined as follows:

$$
\operatorname{Similarity}(s 1, s 2)=\frac{\text { Headers }(s 1) \cap \text { Headers }(s 2)}{\text { Headers }(s 1) \cup \text { Headers }(s 2)}
$$

Here, $s 1$ and $s 2$ represent source files. Headers indicates the set of headers that are included in each source file. A similarity of 1.0 indicates all of the included headers in $s 1$ and $s 2$ are the same.

- Frontend Ratio. To identify whether a source file is frontendintensive or not, we calculate the frontend ratios of the compile time of the source file by using the timer report feature in the compiler (-ftime-report). If the frontend ratio is $1.0(100 \%)$, all compilation tasks of the source file are consumed by the frontend.

- Compile-Time Speedup. Another important metric is by how much unified builds reduce the compile time compared with compiling each source file individually. Here, we can calculate the speedup by dividing the sum of each compile time ( $C T(s 1)$ and $C T(s 2))$ by the compile time of using unified builds (CT(unify)):

$$
\text { Speedup }(s 1, s 2)=\frac{C T(s 1)+C T(s 2)}{C T(\text { unify })}
$$

- Expected Penalty for Incremental Builds. Another metric of unified builds is the expected penalty on incremental builds caused by unified builds. This penalty is defined as the expected value of the overhead of the incremental build assuming that one of the merged source files is updated; here, all source files are updated with the same probability. For example, when two source files are unified, the penalty is computed as

$$
\text { Penalty }=\frac{C T(\text { unify })}{C T(s 1)} \times 0.5+\frac{C T(\text { unify })}{C T(s 2)} \times 0.5
$$

A penalty of 1.0 indicates that unified builds cause no overhead on incremental builds.

\section{RESULTS}

The experiments were conducted on WebKit (git-svn-id:230391). WebKit has three components; JavaScriptCore for the JavaScript engine, WebCore for the rendering engine, and WebKit for the multi-process architecture. Each component uses unified builds independently. Section 4.1, 4.2, 4.3, and 4.4 only show the results for JavaScriptCore; the results for the other components are similar. Section 4.5 evaluates the effectiveness of unified builds in the entire WebKit project.

We used an Intel(R) Xeon(R) 8-core CPU E5-2620 v4 @ 2.10GHz, 128GB of DRAM, and 1TB of HDD. We specified 16 threads as job threads when building the entire WebKit. We used LLVM/Clang (git-svn-id:329912) as the compiler.

\subsection{RQ1: How does the similarity of the headers included among the source files affect unified builds?}

To understand the potential impact of header similarity, we randomly chose a source file from JavaScriptCore, performed unified builds for all other source files, and measured compile-time speedups and expected penalties for incremental builds. We selected API/JSBase. cpp, which is the first source file in alphabetical order. There are 959 other source files that can be bundled with it. We tested all combinations of unified builds with API/JSBase.cpp. 
Figure 4 shows the compile-time speedups and expected penalties. The header similarity between API/JSBase.cpp and the paired source file is indicated on the $\mathrm{x}$-axes. The $\mathrm{y}$-axes show the impact of unified builds, i.e., the compile-time speedup in Figure 4a and expected penalty on incremental builds in Figure 4b.

According to Figure 4a, the higher the similarity of the included headers is, the greater the speedup that unified builds achieve. If the similarity exceeds 0.90 , unified builds achieve a compiletime speedup of 1.85 on average. In the best case where the paired source file is $\mathrm{jit/JITStubRoutine.cpp}$, the compile-time speedup reaches 1.97. This is good news for incremental builds. Figure $4 \mathrm{~b}$ shows the relationship between the header similarities and expected penalties of incremental builds. This figure as well demonstrates that unified builds incur negligible slowdowns in incremental builds when the header similarities are high. In the case of unifying API/JSBase.cpp and jit/JITStubRoutine.cpp, the expected penalty is only 1.02 . These findings tell us that unified builds can achieve high compilation speedups that incur ignorable slowdowns in incremental builds by bundling appropriate source files.

However, we also find cases where the compile-time speedups are not as high as expected even when the header similarities are high. We will discuss these cases in the next section.

Finding 1: Higher header similarity has positive effects on both unified builds and incremental builds. However, there are exceptions that we should avoid.

\subsection{RQ2: What combinations of source files are not suitable for unified builds?}

To better understand the situations where unified builds do not work well, let us investigate the cases in Figure 4 a where the compile-time speedups are not high even though the header similarities are high. For instance, when we perform unified builds for API/JSBase.cpp and parser/Parser.cpp, the compile-time speedup is only 1.15 , despite the header similarity between the two source files is 0.94 . The root cause of this bottleneck is the imbalanced compile times of the source files. The compiler consumes 4.29s for API/JSBase. cpp, but 28.81s for parser/Parser. cpp. Accordingly, the unified build of the two source files takes $28.88 \mathrm{~s}$. This shows that the compile time of unified builds peaks at the compile time of parser/Parser.cpp. It turns out that unified builds between source files with significantly different compile times are not desirable.

In addition, we find that certain source code files are not appropriate for unified builds. Here, since unified builds are adequate for reducing unnecessary header reads, parses, and specializations of templates, it is preferable to apply unified builds to source files that incur a lot of header processing. However, there are source files where the header processing is not dominant, as shown in Figure 2. Applying unified builds to such files is of limited benefit.

To clarify this situation, let us analyze how the characteristics of the compile times of the source files affect the unified builds. The experiment conducted in this case is similar to the one in Figure 4a. We performed the experiment on not only API/JSBase.cpp but also the 959 source files. The analysis entails testing 249,813 bundle combinations in total and calculating the average compile-time speedups for header similarities of more than 0.90 for each source file. However, to consider the imbalance in the compile times of the source files, we will exclude results in which there is a $50 \%$ difference or more in compile time from the analysis, for example, the case of unified builds of API/JSBase. cpp and parser/Parser. cpp where the compile time difference is $571 \%$ (24.52s). Moreover, besides compile-time speedups, we will also estimate the average expected penalty for incremental builds.

Figure 5 shows the results. The $\mathrm{x}$-axis indicates the frontend ratio relative to the entire compile time of each source file. The $y$-axis in Figure $5 \mathrm{a}$ is the average compile-time speedup when applying unified builds to each source file, while the $y$-axis in Figure $5 b$ is the average expected penalty on incremental builds. According to these figures, the frontend ratio is related to the average compile-time speedup and expected penalty on unified builds. As the frontend ratio increases to 1.0, the average compile-time speedup reaches 2.0 almost linearly. Figure $5 \mathrm{~b}$ also indicates that the average expected penalty for incremental builds becomes smaller when applying unified builds to frontend-intensive source files. However, when the frontend ratio is low, the average compile-time speedup and expected penalty increase, resulting in unacceptable slowdowns in incremental builds.

Finding 2.1: We should avoid unified builds for source files that have significantly different compile times.

Finding 2.2: If the source files are frontend-intensive, they are appropriate for unified builds. However, if not, they are not suitable for unified builds, resulting in noticeable incremental build slowdowns.

\subsection{RQ3: Where are the source files that can be unified?}

Currently, WebKit only unifies the source files in the same directory. To better understand the distribution of source files that are suitable for unified builds, let us explore the header similarities and frontend ratios across directories. Figure 6 shows a heat map of expected header similarities of JavaScriptCore. Each label in the figure represents a directory name, and each annotation in a box indicates an expected similarity. We define the expected similarity between two directories as the average header similarity when we perform unified builds on a source file of the directory indicated on the $\mathrm{x}$-axis and another source file of the directory indicated on the y-axis.

According to the figure, there are many directory combinations where the expected header similarities are high; for example, the expected similarity of the combination of API and bindings is 0.90 . This value is as high as when unifying source files only within API. This result indicates that unified builds seem to be effective for source files in different directories.

As Finding 2.2 says, the frontend ratio of the compile time of each source file is an important factor affecting the efficiency of unified builds. To show that the frontend ratio is not an obstacle to unifying source files in different directories, let us examine the distribution of the frontend ratios of each source file across directories. Figure 7 shows the average frontend ratios of each directory. The source 


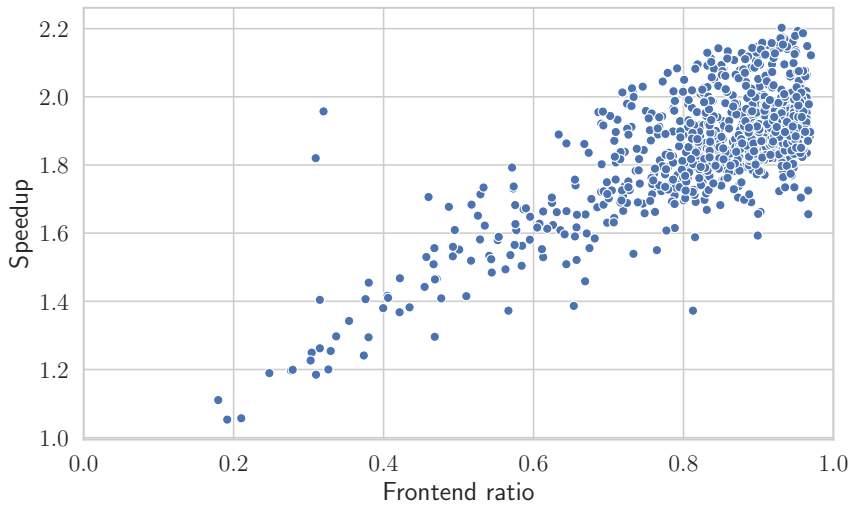

(a) Compile-Time Speedup (higher is better)

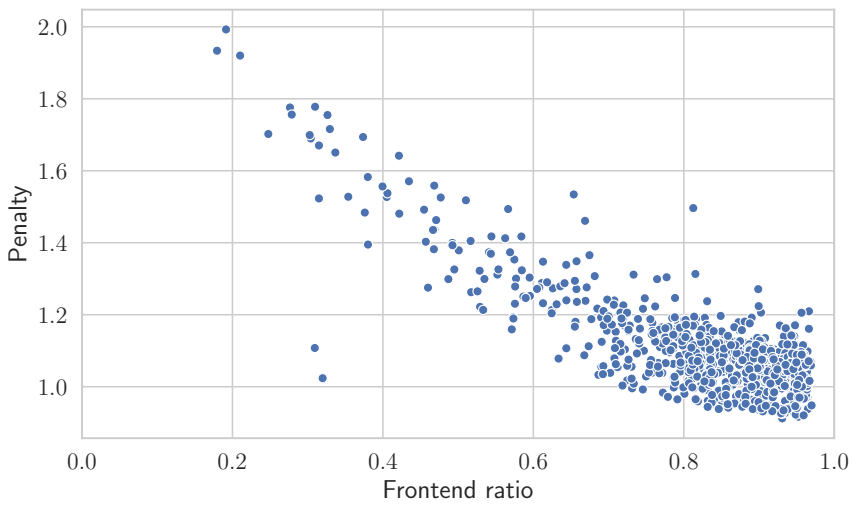

(b) Expected Incremental Builds Penalty (lower is better)

Figure 5. These figures show the impact of the frontend ratio of the compile time of source files on the unified builds. The $x$ axes indicate the frontend ratio. The $y$-axis is the speedup in Figure $5 \mathrm{a}$ and expected penalty in Figure $5 \mathrm{~b}$. The figures indicate that unified builds achieve high speedups and low penalties when the merged source files are highly frontend-intensive

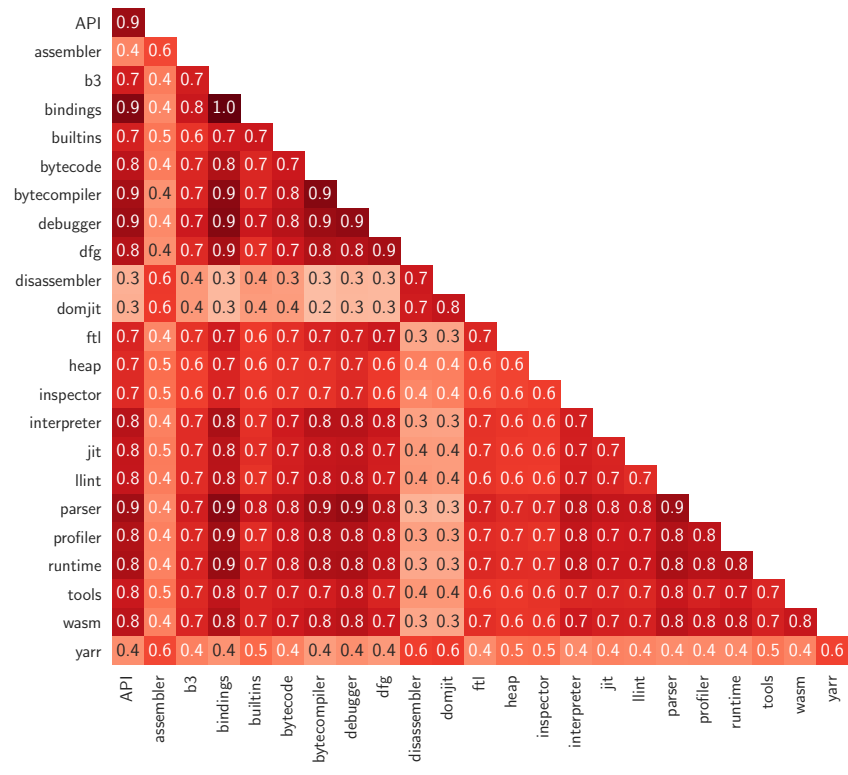

Figure 6. Heat map of header similarity among subdirectories in JavaScriptCore. Each annotation shows the expected similarity when we combine a source file in a subdirectory on the $x$-axis with another in a subdirectory on the $y$-axis. This map indicates that many source files have high similarity to other source files in different directories.

files that are frontend-intensive are evenly distributed. The average frontend ratios exceed 0.7 in 20 out of 23 directories.

Finding 3: We can unify source files not only in the same directory but also in different directories.

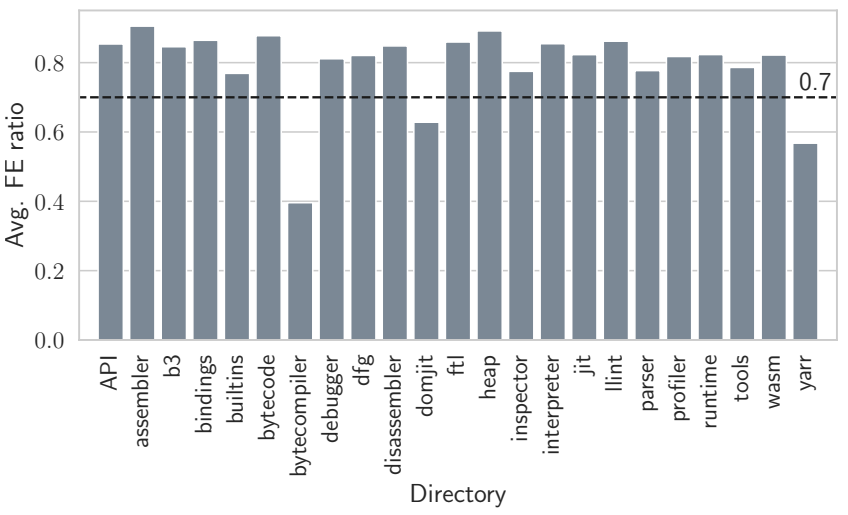

Figure 7. Distribution of source files in JavaScriptCore in terms of the frontend ratio. The directory names are listed on the $x$-axis. The $y$-axis shows the average frontend ratio of the source files that are in each directory. According to the figure, the source files that are frontend-intensive are evenly distributed.

\subsection{RQ4: How many source files should be merged into a unified source file?}

Here, we discuss the number of source files that are bundled into one unified source file. As we described before, unified builds achieve high compile-time speedups and do not cause incremental build slowdowns by bundling appropriate source files; for instance, when applying unified builds to API/JSBase.cpp and jit/JITStubRoutine.cpp, the expected penalty for incremental builds is only 1.02. To see if unified builds scale to the bundle size, we analyze the compile time of unified builds while varying the bundle size from 2 to 32 . Since the frontend ratio of the compile time is related to compile-time speedups and expected penalties of incremental builds, we investigate three source files with different frontend ratios: API/JSBase. cpp, runtime/LiteralParser.cpp, and parser/Parser.cpp with frontend ratios of $0.90,0.52$, and 0.18 , 
Table 1. The table shows the compile times of unified builds (UB) of three source files for bundle sizes from 2 to 32 . The $U B$ (WebKit) column shows the result of WebKit where the bundle size is 8 , and the UB (Manual) column indicates the result of applying our unify rule instead. According to the table, the unified build of JSBase.cpp does not incur large compilation overheads even if the size is larger than that of the current WebKit.

\begin{tabular}{|c|c|c|c|c|c|c|c|c|}
\hline \multirow{2}{*}{ Source file } & \multirow{2}{*}{ Frontend Ratio } & \multirow{2}{*}{ Compile time } & UB (WebKit) & \multicolumn{5}{|c|}{ UB (Manual) } \\
\hline & & & 8 & 2 & 4 & 8 & 16 & 32 \\
\hline JSBase.cpp & 0.90 & 4.26 & 4.62 & 4.36 & 4.43 & 4.46 & 4.61 & 4.97 \\
\hline LiteralParser.cpp & 0.52 & 7.49 & 10.59 & 7.84 & 8.87 & 11.08 & 14.90 & 20.80 \\
\hline Parser.cpp & 0.18 & 28.62 & 42.13 & 57.10 & 84.98 & 124.82 & 183.88 & 307.50 \\
\hline
\end{tabular}

respectively. We manually select the merged source files to maximize compile-time speedups and minimize the expected penalties on incremental builds by referring to the analysis of Figure 5.

Table 1 shows the results. The compile time of unified builds (UB) when using the unify rule of WebKit is shown in UB (WebKit) $U B$ (Manual) indicates the results of manually unifying source files. The compile time of each source file and its frontend ratio are also shown.

According to the table, in the case of JSBase.cpp which is highly frontend-intensive, unified builds scale well with negligible slowdown in incremental builds. For example, when the bundle size is 32 , the slowdown is only 0.71 s relative to the compile time of JSBase.cpp itself. This result suggests that more than eight source files can be unified with JSBase.cpp; eight is the default bundle size of WebKit. However, if the frontend ratio of a source file is low, the slowdown becomes noticeable. In the case of LiteralParser.cpp, the slowdown incurred by unified builds is $13.51 \mathrm{~s}$ when the size is 32. Furthermore, in the case of Parser.cpp, the slowdown reaches 278.88s and becomes unacceptable.

The reason why the compile time of UB (WebKit) is shorter than that of UB (Manual) when the bundle is the same is that incremental build slowdowns are not considered in UB (WebKit). In the unified builds of WebKit, eight source files are automatically merged into one unified source file in alphabetical order. As a result, source files that have a significant difference in compile time become bundled into a unified source file. For example, in the case of Parser.cpp, NodesAnalyzeModule.cpp is merged in the same unified source file in UB (WebKit). The compile time of

NodesAnalyzeModule.cpp is 3.38 s by itself, but unified builds take 42.13 s since eight source files are bundled. Thus, the recompile overhead of NodesAnalyzeModule.cpp is $1146 \%$. To avoid an unacceptable overhead, source files that are not frontend-intensive should not be unified in incremental builds.

Finding 4.1: Unified builds scale as long as the bundled source files are frontend-intensive.

Finding 4.2: Source files that are not frontend-intensive should not be unified in incremental builds.

\subsection{RQ5: How can we improve unified builds (in WebKit)?}

To determine how much unified builds can be improved by using a more sophisticated unification strategy, we wrote a script that
Table 2. Characterization of code modifications for unified builds with the guidance of our script.

\begin{tabular}{lr} 
Description & LOC \\
\hline \hline Add \#includes of required header files & 122 \\
Add using namespace & 4 \\
De-inline functions & 8 \\
Fix ambiguous reference & 95 \\
Fix name conflicts & 53 \\
Fix missing \#pragma once & 1 \\
\hline All & 283
\end{tabular}

automatically generates unified source files meeting the following unify rules:

- Only unify the source files whose header similarities are high (based on Finding 1). In this study, we set 0.9 as the similarity threshold.

- Do not unify the source files that have a significant difference in compile time (based on Finding 2.1). Our script does not bundle source files that have a $50 \%$ difference in compile time.

- Do not unify source files that are not frontend-intensive (based on Finding 2.2 and Finding 4.2). We exclude source files whose frontend ratio is less than 0.2 from the candidates to be unified.

- Bundle source files not only in the same directory, but also in different directories (based on Finding 3).

- When the bundle size is over eight, unify up to eight source files that are not highly frontend-intensive (based on Finding 4.1). Our script specifies 0.6 as the threshold to decide whether a source file is highly frontend-intensive or not.

Note that our script is tailored to the build mechanism of WebKit. WebKit prepares its own scripts and modules for CMake to implement unified builds. Our experiments were conducted by modifying those scripts and modules. In the future, we will generalize our approach to other projects.

We must slightly modify WebKit so that the unified builds are instrumented by our script because it changes the combinations of source files. Although the modification is a problem for unified builds, it is not one peculiar to our approach. Unified builds inherently require code modifications in order to avoid compile errors such as multiple variable declarations. This happens if static variables of the same name are declared in multiple files bundled in a unified file. WebKit developers have to fix the source code every time they encounter this problem [28]. 
To get an idea of the kinds of modification required by our script, Table 2 categorizes 283 code edits. In performing unified builds on various bundle configurations, 122 additional \#includes must be added to the source files because the changes to the bundle combinations eliminated some of the headers that are needed. For the same reason, four lines of code are added for using namespace and eight lines are changed in order to remove inline attributes of functions. Furthermore, some compiler errors incurred by unified builds are fixed. Our script bundles the source files in different directories so that ambiguous references happen when the same named data is defined in different namespaces. To fix these errors, 95 namespace specifications are added. Additionally, 53 code locations are modified to avoid name conflicts across different source files. Such compile errors can be automatically fixed by introducing new supports into compilers for unified builds [19]. Interestingly, we discovered a bug in which one header is not include-guarded. We reported this bug, and our patch was accepted [11].

To investigate the impact of our script on unified builds, let us compare the build efficiencies had when using our scripts and using the unify-rule in WebKit in terms of the number of unified source files, full build time, and incremental build slowdown. To calculate the slowdown of incremental builds, we measure the rebuild time after accessing one source file. We calculate the mean, P90, P99, and the worst incremental build slowdown in each case. The baseline is the rebuild time when unified builds are not used.

Table 3 shows the overall statistics. Note that WebKit refers to unified builds using the default unify rule of WebKit and Guided indicates using unified builds guided by our script. The maximum bundle size is listed in parentheses; e.g., WebKit (8) represents unified builds using the default unify rule of WebKit where up to eight source files in the same directory are bundled into one unified source file. In WebKit (8), 665 unified source files are generated. The full build takes 26 minutes and 21 seconds with 16 threads. The average slowdown of incremental builds that developers reported as the worst case slowdown [22] is $21 \%$. However, the tail slowdown is worse than they expected. The P90 and P99 slowdowns are 58\% and $126 \%$, respectively. In the worst case, the rebuild time goes from 19 s to 110 s (479\%); this case happens when DFGSafepoint.cpp is touched.

Guided (8) shows the results of the unify rule guided by our script specifying a maximum bundle size of eight. The number of created unified source files reaches 861 . Our script outputs more unified source files than the default script of WebKit, because it does not unify source files that are bundled in WebKit to reduce the incremental build slowdown. Surprisingly, both the full build time and incremental build slowdown are reduced. The full build time is reduced by $2.66 \%$, and the slowdowns are reduced in all metrics. In the worst case, the slowdown is $129 \%(24 \mathrm{~s}->55 \mathrm{~s})$ when BuiltinExecutables.cpp is touched. This is because unified builds guided by our script bundle source files in a better way than WebKit (8).

By increasing the bundle size, unified builds guided by our script can reduce the full build time at the expense of slightly increasing the incremental build slowdown. In Guided (16), the full build time is reduced to $23 \mathrm{~m}: 09 \mathrm{~s}$, and the worst slowdown is $140 \%$ (20s $\rightarrow$ $48 \mathrm{~s}$ ) when ObjectRef.cpp is touched. In Guided (32), the full build time is reduced to $22 \mathrm{~m}: 12 \mathrm{~s}$ and the worst slowdown is $145 \%(22 \mathrm{~s}$ $\rightarrow 54 \mathrm{~s}$ ) when BaseCheckableInputType.cpp is touched. The full build times of WebKit (16) and WebKit (32) are shorter than those of Guided (16) and Guided (32). This is because, to reduce the full build times, incremental builds are sacrificed in the rules of WebKit. The worst slowdowns increase to $638 \%$ and $793 \%$ when the maximum bundle sizes are 16 and 32, respectively. This is not fun for developers who actively modify WebKit. Comparing the statistics of WebKit (8) and Guided (32), one sees that unified builds achieve more of a build speedup without significantly slowing down incremental builds. These results highlight the need for quantitative analyses of unified builds in order to devise a sophisticated unification strategy to generate more efficient compiler tasks.

To explore the factors that contribute to the build speedup and incremental build slowdown, the average header similarities and average bundle size of each unified source file are shown in Table 4. According to the table, the average header similarity among the bundled source files is higher in the case of Guided than in the case of WebKit. This is because our script only bundles source files when the header similarity is over 0.9 . This results in more efficient compiler tasks and higher build throughput. We should also investigate the average number of source files that are critical to the incremental build slowdown: according to the table, more source files are bundled into one on average in WebKit. When the maximum unified size is $32,14.67$ source files are bundled at a time on average in WebKit, while 7.76 files are unified in Guided. This result indicates that our script succeeds in reducing the incremental build slowdown by avoiding undesirable unifications that have negative effects on incremental builds.

To understand the performance difference caused by different unification rules, let us evaluate the performance of the binaries generated by unified builds in each unify-rule. Here, we compare four benchmarks provided from BrowserBench.org [1]: Speedmeter2, JetStream, MotionMark, and ARES-6. Speedmeter2 tests a browser's Web application responsiveness. Its benchmark score is the arithmetic mean of how many operations are run per minute. JetStream combines a variety of JavaScript benchmarks. There are 39 benchmarks that cover a variety of advanced workloads. It reports a single score that balances them using the geometric mean. MotionMark is a graphics benchmark and includes nine tests that measure a browserâĂŹs capability to animate complex scenes at a target frame rate. It calculates the geometric mean of all of the testsâÁZ scores into a single score for the run. In these three benchmarks, bigger scores are better. On the other hand, ARES-6 measures the execution time of JavaScript's newest features. It consists of four benchmarks and reports an overall score which is the geometric mean of all of the scores. Smaller scores are better in this benchmark.

Table 5 shows the overall results. It lists performance degradations for Speedmeter2, JetStream, and MotionMark and execution time overhead for ARES-6. The performance of WebKit deteriorates when using our script. For example, when the maximum bundle size is 32 , the performance degradation of Speedmeter 2 is $2.59 \%$ while the execution time overhead of ARES- 6 is $1.31 \%$. This is because unified builds using the unify-rule of WebKit merge more source files into one than when using our script, as shown in Table 4. As a result, the compiler encounters more optimization opportunities when using WebKit's unify rule. 
Table 3. This table shows the efficiency of unified builds with and without guidance by our script. Statistics including the number of unified source files, full build time, and incremental build slowdown are listed.

\begin{tabular}{|c|c|c|c|c|c|c|}
\hline \multirow{2}{*}{ Label (max unified size) } & \multirow{2}{*}{ \# of unified source files } & \multirow{2}{*}{ Full build time $(-\mathrm{j} 16)$} & \multicolumn{4}{|c|}{ Slowdowns of incremental builds } \\
\hline & & & mean & $\mathrm{P} 90$ & P99 & the worst case \\
\hline WebKit (8) & 665 & $26 \mathrm{~m} \mathrm{21s}$ & $21 \%$ & $58 \%$ & $126 \%$ & $479 \%(19 s \rightarrow 110 s)$ \\
\hline Guided (8) & 861 & $25 \mathrm{~m} \mathrm{39s}$ & $5 \%$ & $22 \%$ & $62 \%$ & $129 \%(24 s \rightarrow 55 s)$ \\
\hline WebKit (16) & 416 & $22 \mathrm{~m} \mathrm{47s}$ & $41 \%$ & $91 \%$ & $214 \%$ & $638 \%(16 s \rightarrow 118 s)$ \\
\hline Guided (16) & 651 & $23 \mathrm{~m} \mathrm{09s}$ & $13 \%$ & $41 \%$ & $88 \%$ & $140 \%(20 s \rightarrow 48 s)$ \\
\hline WebKit (32) & 294 & $21 \mathrm{~m} \mathrm{08s}$ & $74 \%$ & $147 \%$ & $300 \%$ & $793 \%(14 s \rightarrow 125 s)$ \\
\hline Guided (32) & 556 & $21 \mathrm{~m} 58 \mathrm{~s}$ & $27 \%$ & $69 \%$ & $124 \%$ & $145 \%(22 s \rightarrow 54 s)$ \\
\hline
\end{tabular}

Table 4. Statistics of unified builds compared with WebKit and Guided. The table shows the average header similarities among the bundled source files and average number of source files bundled into one.

\begin{tabular}{lrr} 
Label & Avg. header similarity & Avg. bundle size \\
\hline \hline WebKit (8) & 0.75 & 6.49 \\
Guided (8) & 0.95 & 5.01 \\
\hline WebKit (16) & 0.75 & 10.37 \\
Guided (16) & 0.94 & 6.63 \\
\hline WebKit (32) & 0.74 & 14.67 \\
Guided (32) & 0.94 & 7.76
\end{tabular}

However, as Table 3 indicates, the slowdown of incremental builds is not considered in the unify-rule of WebKit. Here, linktime optimization (LTO) and whole program optimization (WPO) have become practical $[23,31]$. In LTO and WPO, the optimization boundary is extended to multiple modules so that differences in the unified source files do not affect the optimization. Accordingly, we expect that the performance difference caused by the diversity of unify-rules will disappear after applying these techniques.

Note that we have not yet confirmed the performance results with LTO because of limitations of the Linux-based toolchains. We used a Linux server to build WebKitGTK+ (WebKit built with GTK+ GUI toolkit), which lacks support for the LTO build configuration. Recently, macOS WebKit has started supporting LTO (Xcode version). We hope to try it in the near future.

\section{DISCUSSION}

So far, we have explored the trade-off between unified builds and incremental builds. However, concurrency is also an important metric of unified builds. Developers usually build their projects concurrently depending on the available CPU cores. After applying unified builds, the number of the files that are passed to the compiler is decreased. As a result, unified builds may have a negative effect on parallel builds. Reduction of concurrency is more of a problem when using distributed compilers (e.g., distcc [5], icecc [7], and goma [6]) and when specifying a lot of parallelism (e.g., build with -j100). We will leave the analysis of the trade-off between unified builds and parallelism to future work.

Our work focuses on the case of WebKit. We plan to investigate the characteristics of unified builds for other large software projects such as Chromium and LLVM/Clang. We also plan to create tools that automatically analyze software projects and build unify-rules for the target projects and evaluate these tools on various projects.

\section{RELATED WORK}

To the best of our knowledge, ours is the first survey on the efficiency of unified builds considering slowdown of incremental builds. Our motivations stem from existing techniques that can help with understanding the needs of unified builds; we discuss these techniques below.

Build Systems and Compiler Caching Tools: Build systems (e.g., GNU Make [10], CMake [4], Ninja [12], Meson [13] and so on) are widely viewed as efficient building processes. Traditionally, they provide a capability to achieve incremental builds wherein the source file edits are tracked and only the updated source files are recompiled. Compile caching tools such as CCache [2] and cHash [21] also enhance incremental builds.

However, since software projects have grown in code size, compilers must compile numerous source files, which takes a long time even when the modifications to the code are small. Projects written in $\mathrm{C}++$ especially suffer from long compile times due to redundant header processing. This problem is the motivation behind large projects such as WebKit and Chromium, which are aimed at integrating unified builds into existing build systems. On the other hand, in 2017, the Meson build system [13] added an option that automatically bundles multiple source files into one file [14]. However, it simply merges all the source files into one in each specified project without considering characteristics of source files. Our study is the first to perform an in-depth analysis of unified builds; it reveals the unclear heuristics that are used to decide whether or not to unify source files. We hope that our analysis offers clues to implementing new features for unified builds in future build systems.

Implement new compilers: Another approach to solving the problem is designing new compilers. Zapcc [17] is a caching $\mathrm{C}++$ compiler based on LLVM/Clang [25], designed to perform faster compilations. Zapcc has an in-memory compilation cache in the client-server architecture for remembering all of the compilation information (e.g., ASTs, macro extensions, IRs, and so on) between runs. As a result, Zapcc can reduce redundant header processing, as unified builds do. However, according to the report by a developer of Zapcc [24], it is a heavily modified version of Clang (the difference in code is about $200 \mathrm{~K}$ ) with additional code outside the LLVM/Clang 
Table 5. Performance evaluations of WebKit. Four benchmarks are tested; Speedometer2, JetStream, MotionMark, and ARES-6. For Speedometer2, JetStream, and MotionMark, the overall scores that each benchmark reports are listed. ARES-6 measures the execution time of JavaScript's features and reports the geometric mean of all of the measured times as the overall score. In Speedometer2, JetStream, and MotionMark, higher scores are better. Smaller time is better in ARES-6. The relative performance degradations and execution time overheads are in parentheses.

\begin{tabular}{lrrrr} 
Label (max unified size) & Speedometer2 (Score) & JetStream (Score) & MotionMark (Score) & ARES-6 (Time) \\
\hline \hline WebKit (8) & 67.51 & 200.54 & 167.72 & $28.30 \mathrm{~ms}$ \\
Guided (8) & $66.20(-1.94 \%)$ & $200.08(-0.23 \%)$ & $166.41(-0.78 \%)$ & $28.66 \mathrm{~ms}(+1.27 \%)$ \\
\hline WebKit (16) & 68.10 & 200.90 & 167.02 & $28.49 \mathrm{~ms}$ \\
Guided (16) & $66.61(-2.19 \%)$ & $199.94(-0.48 \%)$ & $166.78(-0.14 \%)$ & $28.70 \mathrm{~ms}(+0.74 \%)$ \\
\hline WebKit (32) & 68.23 & 200.75 & 168.29 & $28.34 \mathrm{~ms}$ \\
Guided (32) & $66.46(-2.59 \%)$ & $199.92(-0.41 \%)$ & $165.81(-1.47 \%)$ & $28.71 \mathrm{~ms}(+1.31 \%)$
\end{tabular}

codebase. By comparison, unified builds do not require any compiler modification.

Redesigning compilers or even introducing new features to make unified builds easier requires careful considerations [19]. The main reason why such considerations become complicated is the lack of quantitative analysis. We hope that the results of our analysis offer clues to making better decisions when implementing new features for unified builds in compilers.

Precompiled Headers: Precompiled headers are an intermediate form of header file that is faster to process in the compile. Various compilers (e.g., Microsoft Visual C and $\mathrm{C}++$, Clang, and GCC) implement them. However, using precompiled headers requires non-trivial changes to a project build, depending on the include patterns. On the other hand, unified builds compile without any changes to the project, except for a trivial refactoring to avoid name conflicts.

C++ Modules: The module system for $\mathrm{C}++[30]$ is designed to deal with the serious impediments to compile-time scalability incurred by header file inclusion. In $\mathrm{C}++$ modules, developers use import instead of the traditional \#include. The import keyword imports a module that is a binary representation of the corresponding header. Since the module is only compiled once, importing the module into a translation unit is a constant-time operation (independent of the module system). To reduce the cost of template specialization, developers can pre-compute commonly used instantiations by exporting explicit instantiations in modules.

Unfortunately, however, $\mathrm{C}++$ modules are not yet standard. They are rarely used and do not support legacy code or macros found in existing $\mathrm{C}++$ code. Modules require significant code refactoring in the bottom-up approach. Unified builds are currently a practical solution to shorten the long compile times of large projects.

$\mathrm{C}++$ modules and unified builds share certain motivations and goals. Both approaches try to reduce redundant compilation tasks caused by headers. However, unified builds have a more general goal, i.e., that of how to generate more efficient compiler tasks and provide them to compilers. The goal will still exist even when $\mathrm{C}++$ modules become standard; for example, unified builds can bundle the source files that import the same modules into one, resulting in a reduction in unnecessary module imports and deserializations. Furthermore, when modules become standardized, unified builds will not be a barrier to introducing modules in existing projects. Expected compile errors such as name collisions among modules have been eliminated in projects that have already adopted unified builds.

Cross-Module Optimizations: Cross-module optimization (CMO) is an effective way to improve runtime performance that works by extending the scope of optimizations across source module boundaries. There are several approaches to $\mathrm{CMO}$, including link-time optimization (LTO) [9, 18, 23, 29], whole program optimization (WPO) [20, 31], and lightweight inter-procedural optimization (LIPO) [26, 27]. One concern about such techniques is the long analysis time for the inter-procedural optimizations. Here, there are designs and implementations for incremental LTO and WPO $[23,31]$ that are as scalable as a regular non-CMO build. When using these techniques with unified builds, differences in performance caused by changing the unify-rule have no effect because the whole program is analyzed and optimized as if it were a single source-level compilation unit.

\section{CONCLUSION}

We have presented the first comprehensive investigation of unified builds. Our in-depth analysis of WebKit has revealed the following:

- A higher header similarity has positive effects both on unified builds and incremental builds.

- Unified builds for source files that have large differences in compile time are not desirable.

- Source files that are not frontend-intensive are not appropriate for unified builds.

- It is possible to unify source files not only in the same directory but also in different directories.

- Unified builds are effective when the merged source files are frontend intensive.

- Source files that are not frontend-intensive should not be unified for incremental builds.

We performed an empirical study on improving the unify-rule in WebKit. When the unify size is up to eight, the unify-rule tuned by us outperforms the original rule of WebKit. The full build time is reduced by $2.66 \%$, and the overhead of incremental builds is also reduced.

We hope that these findings will be of help in deriving a more intelligent strategy of unification and will serve as a basis of discussion on future build systems, compilers, and module systems that cooperatively generate efficient compiler tasks. 


\section{ACKNOWLEDGMENTS}

This work was supported in part by the Japan Science and Technology Agency (JST CREST JPMJCR1683) and the Japan Society for the Promotion of Science (JSPS KAKENHI JP16K00104).

\section{REFERENCES}

[1] [n. d.]. BrowserBench.org - Browser Benchmarks. https://browserbench.org/.

[2] [n. d.]. ccache - Compiler cache. https://ccache.samba.org/.

[3] [n. d.]. The Chromium Projects. http://www.chromium.org/.

[4] [n. d.]. CMake. https://cmake.org/.

[5] [n. d.]. distcc/distcc: distributed builds for C, C++ and Objective C. https: //github.com/distcc/distcc.

[6] [n. d.]. Goma client. https://chromium.googlesource.com/infra/goma/client/.

[7] [n. d.]. icecc/icecream: Distributed compiler with a central scheduler to share build load. https://github.com/icecc/icecream.

[8] [n. d.]. Jumbo / Unity builds. https://chromium.googlesource.com/chromium/ $\mathrm{src} /+/ \mathrm{lkgr} /$ docs/jumbo.md.

[9] [n. d.]. LLVM Link Time Optimization: Design and Implementation. https: //llvm.org/docs/LinkTimeOptimization.html.

[10] [n.d.]. Make - GNU Project - Free Software Foundation. https://www.gnu.org/ software/make/.

[11] [n. d.]. Missing \#pragma once in WasmOpcodeOrigin.h. https://bugs.webkit. org/show_bug.cgi?id=190699.

[12] [n. d.]. Ninja, a small build system with a focus on speed. https://ninja-build.org/.

[13] [n. d.]. The Meson Build system. https://mesonbuild.com/.

[14] [n. d.]. Unity builds. http://mesonbuild.com/Unity-builds.html.

[15] [n. d.]. WebKit. https://webkit.org/.

[16] [n. d.]. WebKit Build Central. https://build.webkit.org/.

[17] [n. d.]. Zapcc - A (Much) Faster C++ Compiler. https://www.zapcc.com/.

[18] Andrew Ayers, Stuart de Jong, John Peyton, and Richard Schooler. 1998. Scalable Cross-Module Optimization. In Proceedings of the ACM SIGPLAN 1998 Conference on Programming Language Design and Implementation (PLDI).

[19] Mostyn Bramley-Moore. [n. d.]. [cfe-dev] JumboSupport: making unity builds easier in Clang. http://lists.llvm.org/pipermail/cfe-dev/2018-April/057579.html.
[20] Preston Briggs, Doug Evans, Brian Grant, Robert Hundt, William Maddox, Diego Novillo, Seongbae Park, David Sehr, Ian Taylor, and Ollie Wild. 2007. WHOPR - Fast and Scalable Whole Program Optimizations in GCC. https: //www.gnu.org/software/gcc/projects/lto/whopr.pdf.

[21] Christian Dietrich, Valentin Rothberg, Ludwig Füracker, Andreas Ziegler, and Daniel Lohmann. 2017. cHash: Detection of Redundant Compilations via AST Hashing. In Proceedings of the 2017 USENIX Annual Technical Conference (ATC).

[22] Geoffrey Garen. 2017. [webkit-dev] Growing tired of long build times? Check out this awesome new way to speed up your build... soon (HINT: It's not buying a new computer). https://lists.webkit.org/pipermail/webkit-dev/2017-August/ 029508.html.

[23] Teresa Johnson, Mehdi Amini, and Xinliang David Li. 2017. ThinLTO: Scalable and incremental LTO. In Proceedings of the 2017 International Symposium on Code Generationand Optimization (CGO).

[24] Yaron Keren. [n. d.]. zapcc compiler. https://lists.llvm.org/pipermail/cfe-dev/ 2015-May/043155.html.

[25] Chris Lattner and Vikram Adve. 2004. LLVM: A Compilation Framework for Lifelong Program Analysis \& Transformation. In Proceedings of the 2004 International Symposium on Code Generation and Optimization (CGO). Palo Alto, California.

[26] Xinliang David Li, Raksit Ashok, and Robert Hundt. [n. d.]. LIPO - Profile Feedback Based Lightweight IPO. https://gcc.gnu.org/wiki/LightweightIpo.

[27] Xinliang David Li, Raksit Ashok, and Robert Hundt. 2010. Lightweight FeedbackDirected Cross-Module Optimization. In Proceedings of the 2010 International Symposium on Code Generation and Optimization (CGO).

[28] Keith Miller. 2017. [webkit-dev] Unified source builds: A new rule for static variables. https://lists.webkit.org/pipermail/webkit-dev/2017-August/029465.html.

[29] Sungdo Moon, Xinliang D. Li, Robert Hundt, Dhruva R. Chakrabarti, Luis A. Lozano, Uma Srinivasan, and Shin-Ming Liu. 2004. SYZYGY - A Framework for Scalable Cross-Module IPO. In Proceedings of the 2004 International Symposium on Code Generation and Optimization (CGO).

[30] Gabriel Dos Reis, Mark Hall, and Gor Nishanov. 2016. A Module System for C++ (Revision 4). http://www.open-std.org/jtc1/sc22/wg21/docs/papers/2016/ p0142r0.pdf.

[31] Patrick W. Sathyanathan, Wenlei He, and Ten H. Tzen. 2017. Incremental Whole Program Optimization and Compilation. In Proceedings of the 2017 International Symposium on Code Generationand Optimization (CGO). 